\title{
Spectral broadening in femtosecond laser written waveguides in chalcogenide glass
}

\author{
Mark A. Hughes, ${ }^{1, *}$ Weijia Yang, ${ }^{2}$ and Daniel W. Hewak ${ }^{3}$ \\ ${ }^{1}$ Toyota Technological Institute, 2-12-1, Hisakata, Tempaku, Nagoya 468-8511, Japan \\ ${ }^{2}$ Université de Paris Sud 11, Institut de Chimie Moléculaire et des Matériaux d'Orsay, \\ Laboratoire de Physico-Chimie de L'Etat Solide, UMR CNRS-UPS 8182, Bât. 410, 91405 Orsay Cedex, France \\ ${ }^{3}$ Optoelectronics Research Centre, University of Southampton, Southampton SO17 1BJ, United Kingdom \\ *Corresponding author: mark@toyota-ti.ac.jp
}

Received December 3, 2008; revised May 15, 2009; accepted May 15, 2009; posted May 20, 2009 (Doc. ID 104754); published June 15, 2009

\begin{abstract}
Nonlinear spectral broadening to $200 \mathrm{~nm}$, from an initial width of $50 \mathrm{~nm}$, has been demonstrated in gallium lanthanum sulphide glass waveguides from $1540 \mathrm{~nm}, 200 \mathrm{fs}$ pulses at $30 \mathrm{~nJ} / \mathrm{pulse}$. A formation mechanism is presented for these femtosecond laser written waveguides, based on optical characterization and comparisons to previous work. Two different types of waveguide are identified. One has a characteristic long narrow structure and is formed through filamentation caused by self-focusing. The other has a characteristic "teardrop" structure, which is formed by a type IIA photosensitivity mechanism and cumulative heating of glass around a central laser-exposed region. (c) 2009 Optical Society of America

OCIS codes: $320.7110,220.4000$.
\end{abstract}

\section{INTRODUCTION}

Femtosecond (fs) lasers have several advantages over conventional laser systems for the microstructuring of transparent dielectrics. These include the reduction of collateral damage [1] and the ability to achieve subdiffraction limited ablation [2-4]. In gold films, $800 \mathrm{~nm}$ fs lasers have been used to ablate holes roughly $10 \%$ of the focus spot size [5]. This effect was attributed to the minimized thermal diffusion time of ultrashort pulses that have a peak laser fluence slightly above a well-defined ablation threshold [5]. Femtosecond laser modification of transparent solids has many potential applications. The possibility of writing active devices has been demonstrated in $\mathrm{Er}-\mathrm{Yb}$ doped silicate glass [6]. Bulk modification by tightly focused fs lasers has been demonstrated as a tool for threedimensional (3D) optical memory with data storage capacities of up to $10^{16} \mathrm{bits} / \mathrm{m}^{3}$ [7]. Tightly focused fs lasers have also been used for microstructuring in transparent dielectrics [8,9]. Infrared fs laser pulses have been used to permanently photoreduce $\mathrm{Eu}^{3+}$ to $\mathrm{Eu}^{2+}$ in fluorozirconate glass [10]. Photo-oxidation of $\mathrm{Mn}^{2+}$ to $\mathrm{Mn}^{3+}$ has also been observed in silicate glass [9].

Highly nonlinear glass is an excellent candidate material for optical, ultrafast nonlinear devices such as demultiplexers [11], wavelength converters [12], and optical Kerr shutters [13]. This is because of its ability to induce nonlinear phase shifts over much shorter interaction lengths than conventional silica-based devices. In these materials, various waveguiding structures such as fibers, proton beam written waveguides, continuous wave (cw) laser written waveguides, and fs laser written waveguides could be used to realize such devices. Among these, fs laser writing is particularly attractive because, as well as having rapid processing times, waveguiding structures can be formed below the surface of the glass enabling 3D structures to be fabricated. Optical components such as a Fresnel zone plate [14] and a fiber attenuator [9] have been fabricated using fs laser pulses. Several studies have described the fabrication and characterization of waveguides using focused fs laser pulses in phosphate glass [15], chalcogenide glass [16], and heavy metal oxide glass [17]. Of these, chalcogenide glasses are especially attractive because they have a higher nonlinear refractive index and enhanced IR transmission coupled with low maximum phonon energy. The nonlinear refractive index of chalcogenide glasses is, in general, higher than oxide glasses with the same linear refractive index. This is believed to be a consequence of the large polarizability of the chalcogen ions [18]. Of the chalcogenide glasses, gallium lanthanum sulphide (GLS) is probably the most notable with respect to optical nonlinear devices as it has the highest nonlinear figure of merit (FOM) of any glass reported to date [19]. In this work the FOM is defined as $\mathrm{FOM}=n_{2} /(2 \beta \lambda)$, where $\lambda$ is the wavelength, $n_{2}$ is the real part of the nonlinear refractive index, and $\beta$ is the two-photon absorption (TPA) coefficient. These parameters are given in Table 1.

In previous work, we reported initial results on fs laser written waveguides in GLS glass [20]. In this work, we report details on the fabrication and characterization of these waveguides and give a more complete discussion of the waveguide formation mechanism. We also report the spectral broadening of a $1540 \mathrm{~nm} 200 \mathrm{fs}$ laser beam in these waveguides.

\section{EXPERIMENTAL}

A. Glass Melting

Samples of GLS were prepared by mixing $65 \%$ gallium sulphide, $30 \%$ lanthanum sulphide, and 5\% lanthanum 
Table 1. Parameters for GLS and GLS Waveguides ${ }^{a}$

\begin{tabular}{lcccc}
\hline & $\begin{array}{c}n_{2} \\
n_{0}\end{array}$ & $\begin{array}{c}\beta \\
(\mathrm{cm} / \mathrm{GW})\end{array}$ & FOM & $\begin{array}{c}\text { Bandgap } \\
(\mathrm{nm})\end{array}$ \\
\hline 2.41 & 2.16 & $<0.01$ & $>7.1$ & 500 \\
\hline
\end{tabular}

${ }^{a} n_{0}, n_{2}$ and $\beta$ were measured at $1.5 \mu \mathrm{m}$ and taken from [19].

oxide (\% molar). Batching was carried out in a drynitrogen purged glove box. Melt components were batched into vitreous carbon crucibles and weighed using a balance with a resolution of $0.001 \mathrm{~g}$. Batches were then transferred to a furnace using a custom-built, closedatmosphere transfer pod. The lanthanum oxide was purchased commercially and used without further purification. The glass was melted at $1150^{\circ} \mathrm{C}$ for around $24 \mathrm{~h}$, in a silica tube furnace, with an initial ramp rate of $20^{\circ} \mathrm{C} \mathrm{m^{-1 }}$ and under a constant argon atmosphere (flow of $200 \mathrm{ml} \mathrm{min}^{-1}$ ). This method was chosen in favor of the sealed ampoule method because volatile impurities such as $\mathrm{OH}^{-}$are carried downstream away from the melt and also because of safety concerns of the ampoules exploding. The melt was rapidly quenched to form a glass by pushing the crucible holder into a silica water jacket. The quenching process is designed to prevent crystallization of the glass by rapidly increasing the viscosity of the glass through rapid temperature drop, hence arresting the nucleation and growth of crystals. The glass was then annealed at $500^{\circ} \mathrm{C}$ for $12 \mathrm{~h}$.

\section{B. Waveguide Fabrication and Characterization}

To write the waveguides, a Ti:sapphire laser (Coherent RegA 9000) emitting a train of pulses with a duration of $150 \mathrm{fs}$, a repetition rate of $250 \mathrm{KHz}$ and a central wavelength of $800 \mathrm{~nm}$ was used. Pulse energy was varied using a variable neutral density filter. The laser beam was focused via a $50 \times$ objective $(\mathrm{NA}=0.55)$ at 100 or $400 \mu \mathrm{m}$ below the surface of the sample and had a focus spot diameter of around $2 \mu \mathrm{m}$. The sample was mounted on a computer-controlled linear motor translation stage, which could move over three axes with a resolution of $20 \mathrm{~nm}$. The propagation direction of the beam used to write the waveguides was in the $+z$ direction of Fig. 1 , and the waveguides were formed by translating the sample along the $x$ axis with various pulse energies and translation velocities. The writing beam was polarized along the waveguide axis ( $x$ axis of Fig. 1). After processing, the end faces of the sample were polished for subsequent characterization.

We wrote two sets of waveguides: one at a focus depth of $\sim 400 \mu \mathrm{m}$ at various pulse energies between 0.21 and $1.75 \mu \mathrm{J} /$ pulse and a translation speed of $200 \mu \mathrm{m} / \mathrm{s}$ (set 1). The other was at a focus depth of $\sim 100 \mu \mathrm{m}$ at various pulse energies between 0.12 and $0.4 \mu \mathrm{J} /$ pulse and translation speeds of 50,100 , and $200 \mu \mathrm{m} / \mathrm{s}$ (set 2). All waveguides were $12 \mathrm{~mm}$ in length. To obtain guided mode profiles, a vertically polarized $633 \mathrm{~nm}$ HeNe laser was coupled into and out of the waveguides with 10 $\times 0.25 \mathrm{NA}$ objectives. The low magnification was needed because of the large mode size of some of the waveguides. The polarization direction was changed with a half-wave plate. The near-field image was then captured by a charged coupled device (CCD) camera.

The refractive index change $(\Delta n)$ profile was deduced from a quantitative phase image, taken in the axis the waveguides were written (the $z$ axis of Fig. 1), using quantitative phase microscopy (QPM) [21]. The QPM system incorporated a PI nanofocusing $\mathrm{Z}$ drive to take infocus and very slightly positively and negatively defocused images; the commercial software (iatia) was then used to calculate the phase image.

Waveguide transmission spectra were taken by coupling a white-light source into the waveguides and dispersing the output from the waveguides in a monochro-

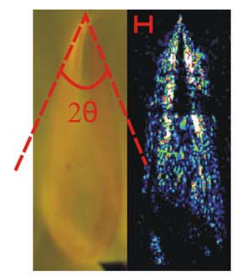

(a)

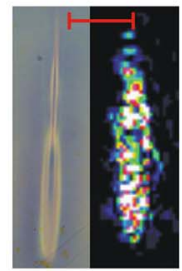

(d)

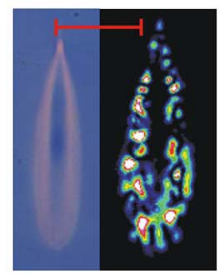

(g)

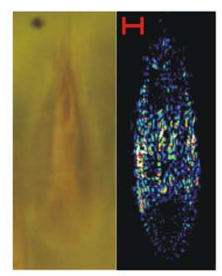

(b)

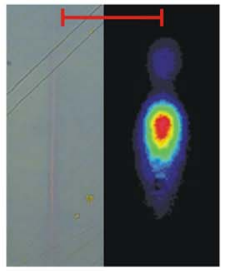

(e)

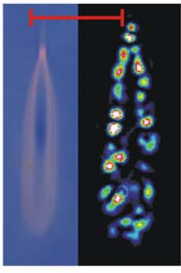

(h)

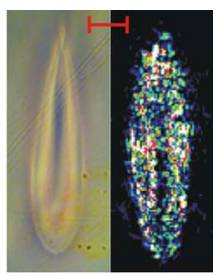

(c)

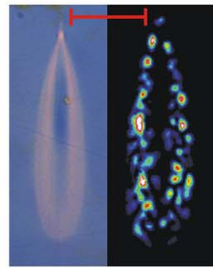

(f)

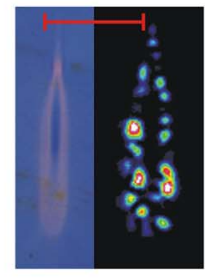

(i)
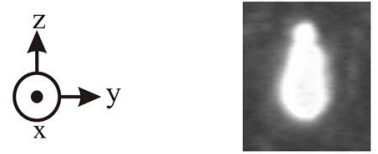

(j)

Fig. 1. (Color online) Waveguide end face transmission optical micrographs (left panel) and near-field guided mode at $633 \mathrm{~nm}$ (right panel). All waveguides were written at a translation speed of $200 \mu \mathrm{m} / \mathrm{s}$. Waveguides (a) to (e) were written at a focus depth of $\sim 400 \mu \mathrm{m}$ (set 1), waveguides (f) to (i) were written at a focus depth of $\sim 100 \mu \mathrm{m}$ (set 2 ). The pulse energies ( $\mu \mathrm{J} /$ pulse) used to write each respective waveguide were $1.75(\mathrm{a}), 1.26(\mathrm{~b}), 0.84(\mathrm{c})$, 0.42 (d), 0.21 (e), 0.40 (f), 0.36 (g), 0.32 (h), and 0.28 (i). (j) shows the guided mode at $1550 \mathrm{~nm}$ of the waveguide in (a). The scale bars shows $20 \mu \mathrm{m}$. 
mator and detecting with a silicon detector using standard phase-sensitive detection. Raman spectra were taken with a Renishaw Ramanscope using a $10 \mathrm{~mW}$ $633 \mathrm{~nm}$ HeNe laser.

To take spectral broadening measurements, the output of the Coherent RegA 9000 was coupled into a Coherent optical parametric amplifier (OPA) 9800, which was tuned to $1540 \mathrm{~nm}$, producing pulses with duration of around $200 \mathrm{fs}$. The pulse energy of the output from the OPA was varied with a variable neutral density filter, and the average power was measured with a Coherent Powermax PM10 thermal power meter head. The beam was coupled into the waveguides with 0.1 or 0.25 NA objective lenses and then coupled out of the waveguides with a $0.25 \mathrm{NA}$ objective lens. The guided modes were isolated with an iris then coupled into a single-mode silica fiber with a $0.1 \mathrm{NA}$ lens and a 0.25 NA objective lens. The beam was then detected with an Ando AQ6315A optical spectrum analyzer (OSA), which had a detection range of 350 to $1750 \mathrm{~nm}$ and was set to a resolution of $5 \mathrm{~nm}$.

\section{RESULTS AND DISCUSSION}

\section{A. Guided Mode Profile and Micrographs}

Figure 1 shows the transmission optical micrograph and near-field-mode profile of waveguides written in GLS glass with pulse energies of $0.21-1.75 \mu \mathrm{J}$ and a scan speed of $200 \mu \mathrm{m} / \mathrm{s}$, with the focus spot either $100 \mu \mathrm{m}$ (set 2) or $400 \mu \mathrm{m}$ (set 1) below the surface of the sample. The figure shows that the cross-sectional shape and size of the waveguides has a strong dependence on the writing pulse energy. The $800 \mathrm{~nm}$ wavelength of the laser used to write the waveguides is within the transmission window of GLS, see Fig. 4, and there is no noticeable material modification occurring until the beam reaches its focus inside the glass. This indicates that a nonlinear absorption process is responsible for waveguide formation. We noted that there was no apparent ablation or cracking of the glass even at the highest pulse energy used of $1.75 \mu \mathrm{J}$, which is $\sim 14$ times the lowest pulse energy $(0.12 \mu \mathrm{J})$ at which material modification was observed (no guided mode could be found for this waveguide).

By examining Figs. 1(a)-1(e) we can describe how the form of the waveguides varies with pulse energy: at pulse energies of $0.84-1.75 \mu \mathrm{J}$ the waveguides have a distinctive "teardrop" shape with a dark central region and a brighter structure surrounding it; this type of waveguide is now referred to as A-type. Similarly shaped crosssections were observed in fs-laser written waveguides in $\mathrm{GeS}_{2}-\mathrm{Ga}_{2} \mathrm{~S}_{3}-\mathrm{CsI}$ glass [22]. At pulse energies of $0.21 \mu \mathrm{J}$ the waveguide structure is very different from the A-type waveguides and is characterized by a long and narrow filamentlike structure with no resolvable dark central region; this type of waveguide is now referred to as B-type. The waveguide written at pulse energies of $0.42 \mu \mathrm{J}$ appears to show a transitional form with characteristics of both A-type and B-type waveguides. The waveguides in Figs. 1(f)-1(i) are all A-type waveguides and were written at a depth of $\sim 100 \mu \mathrm{m}$ below the surface of the sample. These also show a teardrop shape and have a dark central region, which does not appear to guide light (now referred to as region 1) and a brighter structure surrounding it (now referred to as region 2); however, this differentiation of structure is clearer than for the waveguides written at a focus depth of $400 \mu \mathrm{m}$. Examination the guided mode of all the waveguides indicates that, in the waveguides written at a depth of $100 \mu \mathrm{m}$, region 1 does not actively guide light and only region 2 guides light; however, in the waveguides written at a depth of $400 \mu \mathrm{m}$ almost the entire waveguide structure can actively guide light. A small bright spot is visible at the top of the A-type waveguides, especially in Figs. 1(f)-1(i). The position of these spots remained parallel with the face that the writing beam entered from. This indicates they are at the focus spot and that most of the laser energy was deposited before the focus. This explains the asymmetry of the waveguides in the $y$ axis because the waveguides appear to follow the shape of the focal cone. Rotating the polarization of the guided $633 \mathrm{~nm}$ beam from vertical (E field along major axis of waveguide) to horizontal increased the transmitted power by $\sim 10 \%$.

\section{B. Refractive Index Change Profile}

Refractive index change $(\Delta n)$ profile measurements were calculated from the phase change induced by the waveguide structure on light traveling in the $-z$ direction as shown in Fig. 1. They therefore represent an index change cross-section along the $y$ axis induced by the entire waveguide structure along the $z$ axis.

Figure 2 shows the $\Delta n$ profile as a function of writing pulse energy for the set 2 waveguides, which were written at a depth of $100 \mu \mathrm{m}$. The figure shows a double-peak structure with a trough at the center for waveguides written at pulse energies of 0.36 and $0.4 \mu \mathrm{J}$. This double-peak structure indicates that region 1 has undergone a lower refractive index change than region 2 . Since the index change measurement represents the index change induced by all of the waveguide structure along the $z$ axis it remains a possibility that region 1 has undergone a nega-

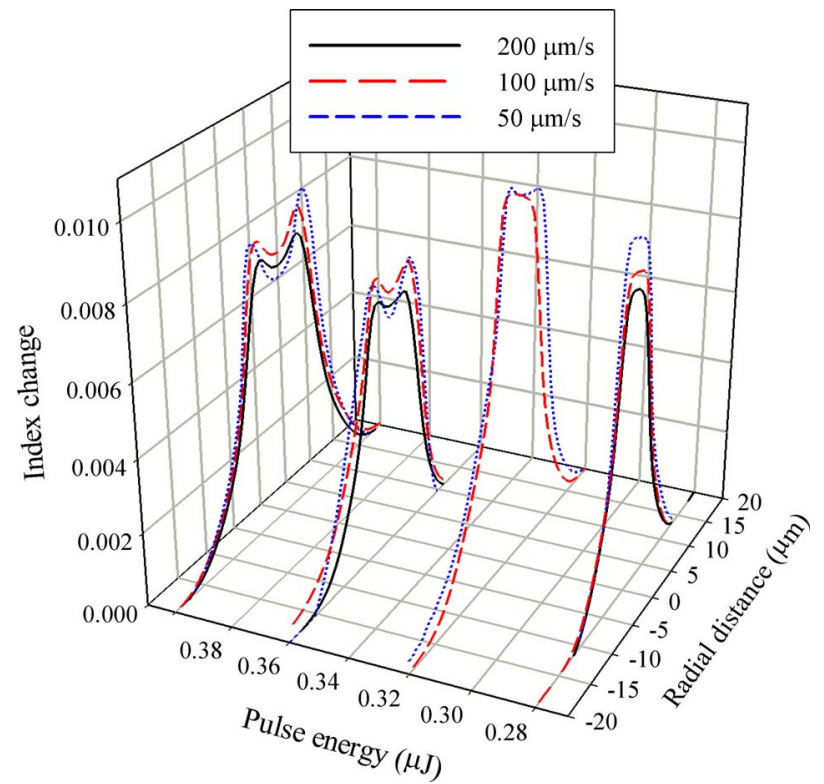

Fig. 2. (Color online) Refractive index change profile as a function of writing pulse energy for set 2 waveguides written into GLS glass at a depth of $\sim 100 \mu \mathrm{m}$ at translation speeds of 50 , 100 , and $200 \mu \mathrm{m} / \mathrm{s}$. 
tive index change. In previous work we reported that region 1 has a lower reflectivity than the surrounding glass [20], indicating it has undergone a negative index change. Region 2 had a higher reflectivity and guided light, indicating it had undergone a positive index change.

Figure 2 shows that, at pulse energies of $0.32 \mu \mathrm{J}$, the double-peak structure of the index change profile is no longer apparent. Examining the micrographs in Figs. 1(f)-1(i) indicates that the disappearance of the doublepeak structure could be due to a reduction in the relative size of region 1 , which has a lower index change than region 2. The increase in maximum index change, as the pulse energy decreases from 0.36 to $0.32 \mu \mathrm{J}$, is also attributed to this effect. The $\Delta n$ may also have saturated, as happens in fs-laser written waveguides in fused silica where the index change for a translation speed of $10 \mu \mathrm{m} / \mathrm{s}$ saturated at $\sim 0.75 \mu \mathrm{J}$; for borosilicate glass the corresponding value was $0.5 \mu \mathrm{J}$ [23]. Figure 2 also shows that reducing the translation speed increases the contrast of the double-peak structure, for example, for waveguides written at $0.4 \mu \mathrm{J} /$ pulse the ratio of the double peaks to the central trough is $1.07,1.11$, and 1.29 for translation speeds of 200,100 , and $50 \mu \mathrm{m}$ respectively, which indicates that region 1 is larger and/or has a lower refractive index in waveguides written with lower translation speeds.

Figure 3 shows the $\Delta n$ profile as a function of writing pulse energy for set 1 waveguides that were written at a depth of $\sim 400 \mu \mathrm{m}$. Similarly to Fig. 2 there is a deviation from a double-peak structure at $0.42 \mu \mathrm{J} /$ pulse; however, examining the micrograph of this waveguide in Fig. 1(d) indicates that this originates from a transition from A-type to B-type waveguides. Figures 2 and 3 show the index change appears to reach a maximum of $\sim 0.01$ at $0.32 \mu \mathrm{J} / \mathrm{pulse}$ and $0.84 \mu \mathrm{J} / \mathrm{pulse}$ for waveguides written at a depth of 100 and $400 \mu \mathrm{m}$, respectively. This nonmonotonic increase is attributed to a saturation of the index change and an increase of the relative size of region 1

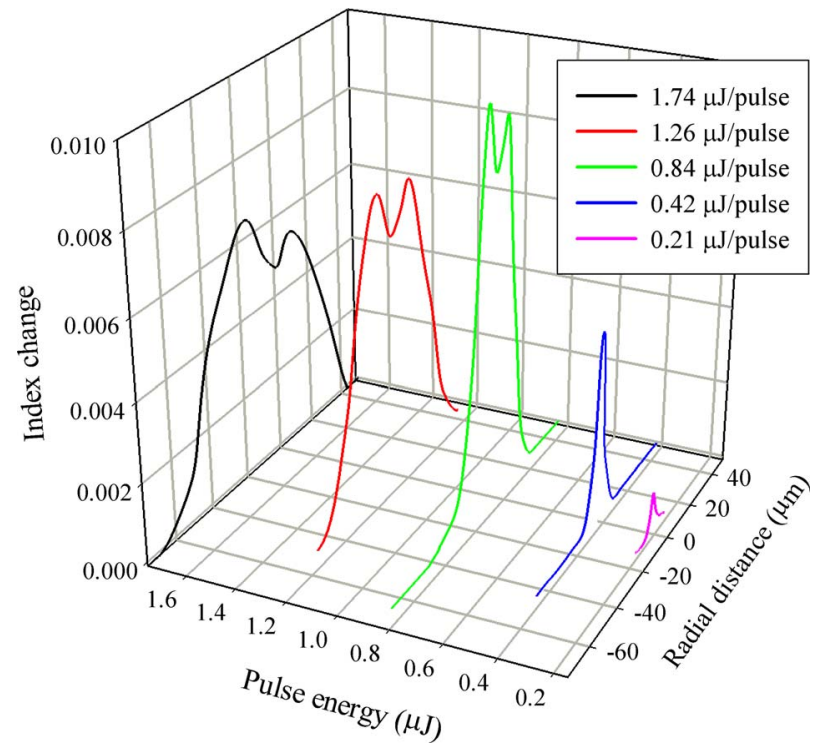

Fig. 3. (Color online) Refractive index change profile as a function of writing pulse energy for set 1 waveguides written into GLS glass at a depth of $\sim 400 \mu \mathrm{m}$ at a translation speed of $200 \mu \mathrm{m} / \mathrm{s}$. with increasing pulse energy. This maximum observed index change compares to 0.08 in $\mathrm{As}_{2} \mathrm{~S}_{3}$ glass [24], 3.5 $\times 10^{-3}$ in silica glass [23] and $4 \times 10^{-3}$ in borosilicate glass [23].

\section{Transmission and Micro-Raman Measurements}

Figure 4 shows the transmission spectra through $12 \mathrm{~mm}$ of bulk GLS and set 1 waveguides written at various pulse energies. When viewed in units of absorbance it is clearer to see that the band edge of GLS lies at around $500 \mathrm{~nm}$ [25]; however, units of transmission make the band edge shifts more apparent. Other authors have suggested a color-center model to explain index modification in glasses after fs-laser exposure [3,23,26]. This model stipulates that fs-laser pulses introduce color centers in sufficient numbers and strength to alter the index through a Kramers-Kronig mechanism. Figure 4 shows that all of the waveguides have an Urbach edge red shift of around $15 \mathrm{~nm}$, and all except the waveguide written at $0.21 \mu \mathrm{J} /$ pulse have a band edge red shift of around $15 \mathrm{~nm}$. Femtosecond laser written waveguides in $\mathrm{As}_{40} \mathrm{~S}_{60}$ glass also showed a $15 \mathrm{~nm}$ red shift of the band edge [16]. This was related by the Kramers-Kronig relation to a refractive index increase of $5 \times 10^{-4}$. The band edge and refractive index of $\mathrm{As}_{40} \mathrm{~S}_{60}$ glass are approximately the same as GLS [19]. Therefore the magnitude of the index change in the GLS waveguides in Fig. 3 that could be attributed to the Kramers-Kronig processes is expected to be approximately $5 \times 10^{-4}$. This is a factor of 20 smaller than the index change we measured by QPM in Fig. 3. This indicates that color-center formation does not play a significant roll in the index modification of fs-laser written GLS waveguides.

The red shift of the band edge, otherwise known as photodarkening, is a photoinduced effect commonly observed in chalcogenides. Photodarkening has been studied extensively in $\mathrm{As}_{2} \mathrm{~S}_{3}$ [27-32] but relatively little in GLS; however, the thermomechanical and optical properties of GLS and $\mathrm{As}_{2} \mathrm{~S}_{3}$ are similar. Several mechanisms have been proposed for photodarkening in chalcogenides including a decrease in average intermolecular distance [31] and photoinduced changes in polarizability of van der Waals

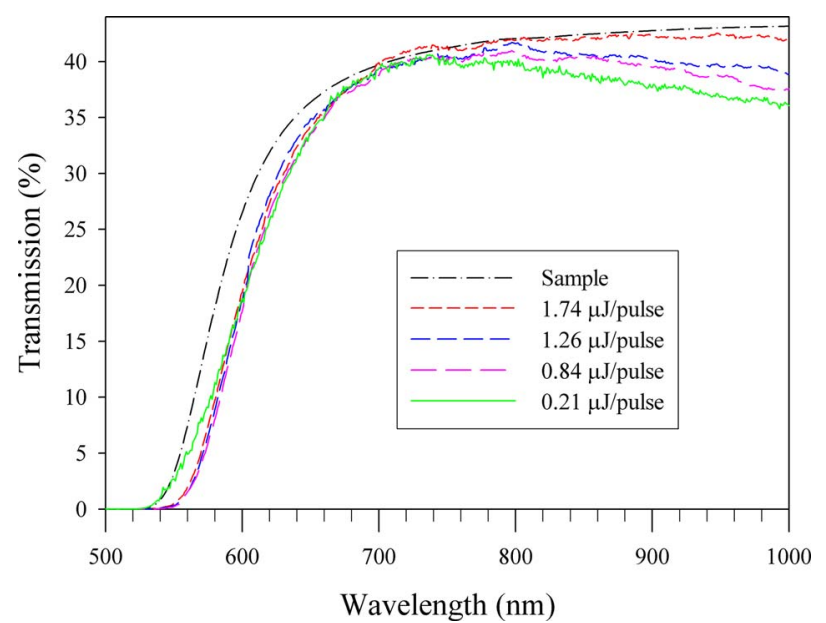

Fig. 4. (Color online) Transmission spectra of GLS sample and set 1 waveguides written at various pulse energies at a depth of $\sim 400 \mu \mathrm{m}$ and a translation speed of $200 \mu \mathrm{m} / \mathrm{s}$. 
type hyperpolarizable bonds [32]. However, we aspire to a more detailed and unified model proposed by Elliott [27]. This states that red shifts of the Urbach edge are related to the breaking and weakening of bonds between nonbonded chalcogen atoms in neighboring layers (intermolecular bond breaking). Also, red shifts of the band edge are related to the breaking of primary covalent bonds followed by the formation of new bonds (intramolecular bond breaking). Figure 4 shows a red shift in both the Urbach edge and band edge for waveguides written at 1.74 to $0.84 \mu \mathrm{J} /$ pulse (A-type waveguides). Therefore we propose that, in A-type waveguides, both intermolecular and intramolecular bond breaking occurs. However, at $0.21 \mu \mathrm{J} / \mathrm{pulse}$ (B-type waveguide) only a red shift in the Urbach edge was observed; therefore we propose that in B-type waveguides only intermolecular bond breaking occurs.

Figure 5 shows micro-Raman measurements of region 1 and 2 of waveguides written at pulse energies of $1.75 \mu \mathrm{J}$ and $1.26 \mu \mathrm{J}$ and two regions of unexposed glass. The top image shows the position at which each micro-Raman spectrum was taken. The insets show an expansion of two regions of interest. Figure 5 shows that the variations in Raman spectra between the waveguide structures and the bulk glass are no greater than the variations between two regions of unexposed glass. The Boson peak could not be observed in these Raman spectra. The broad bands of the Raman spectrum of GLS make structural variations difficult to distinguish. We therefore propose that the molecular bond breaking, indicated by the photodarkening, either doesn't occur in sufficient quantity to be detected
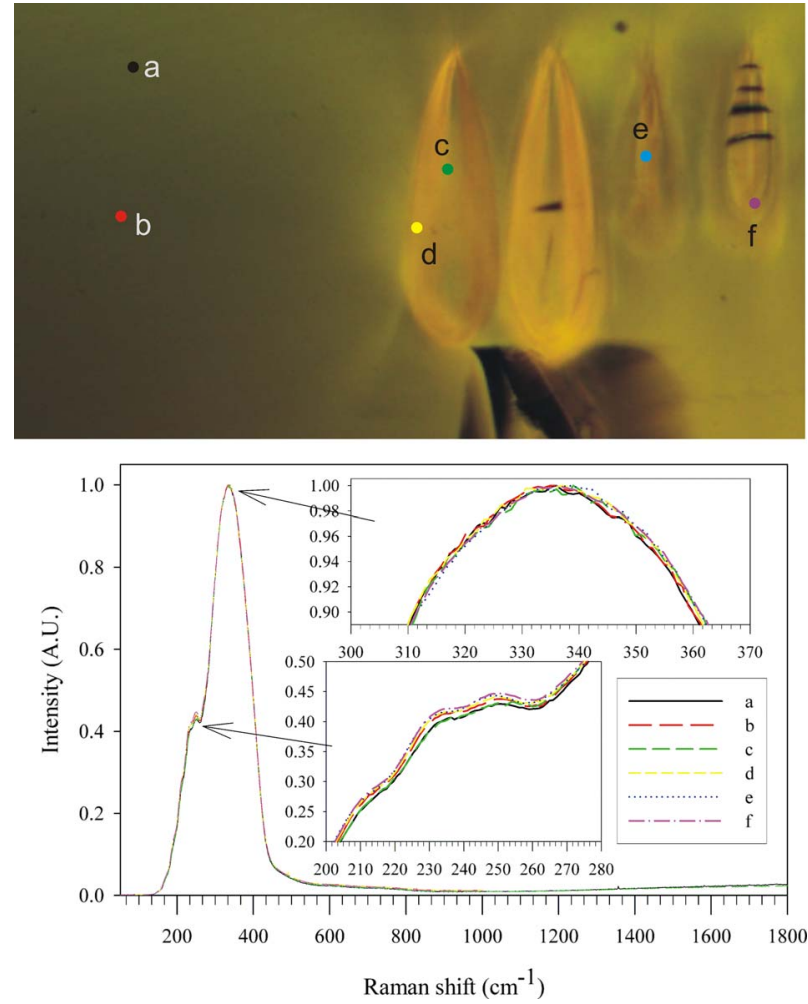

Fig. 5. (Color online) Micro-Raman spectra of set 1 waveguides written at pulse energies of 1.75 and $1.26 \mu \mathrm{J}$ and two regions of unexposed glass. The top image shows the position at which each Raman spectrum was taken. by Raman or the formation of new bonds, which follows the initial breaking of primary covalent bonds, doesn't result in an overall change in structure that can be detected by Raman. Although differences in Raman spectra between waveguide structures and bulk glass have been observed in glasses such as $\mathrm{As}_{2} \mathrm{~S}_{3}$ [24] and aluminosilicate [33], in $\mathrm{PbO}: \mathrm{Bi}_{2} \mathrm{O}_{3}: \mathrm{Ga}_{2} \mathrm{O}_{3}: \mathrm{GeO}_{2}$ glass no differences were observed [17]. Electron dispersive X-ray (EDX) measurements of regions 1 and 2, and the surrounding glass found no compositional variation between these regions greater than the system detection limit of around $1 \%$.

\section{Waveguide Formation Mechanism}

The morphology of the A-type waveguides is rather complex-consisting of a central core (region 1) that has undergone a negative index change and surrounding shell (region 2) that has undergone a positive index change. Comparing this morphology to that of fs waveguides in other glasses yields a close similarity with IOG1 phosphate glass [15], which has similar thermomechanical properties to GLS. The IOG1 waveguides consisted of a central exposed region that had undergone a negative index change and did not guide light. This was surrounded by an unexposed waveguiding region that had undergone a positive index change [15]. The photosensitivity mechanism of UV exposed glasses is often categorized as type I, II, or IIA. Using this classification scheme, our waveguides most closely match the type IIA mechanism because of the nonmonotonic increase in refractive index with exposure dose and the coexistence of a positive and negative index change.

We will now examine three mechanisms that could account for the complex morphology of A-type waveguides: (i) a type IIA photosensitivity behavior in which the sign of the index change can depend on exposure dose, (ii) microexplosions, which result in a rarefied core and compacted shell, (iii) modification by thermal processes caused by cumulative heating.

Type IIA photosensitivity behavior could play part in the formation mechanism of GLS waveguides. In UVexposed germanosilica glass, increasing fluence produced at first a positive index change, then at higher fluence it produced a negative index change [34]. A type IIA photosensitivity mechanism has also been proposed for UVexposed IOG1 phosphate glass [35]. If a similar mechanism occurs in fs-exposed GLS we can envisage region 1 being formed by the higher intensity at the center of the writing beam, the sides of region 2 being formed by lower intensities at the periphery of the beam, the bottom when the beam is far from its focus, and the top after most of the beam has been absorbed. To establish if it is possible that direct exposure to the focal cone of the writing beam could form the waveguide in Fig. 1(a), we measured the half-angle $(\theta)$ of this waveguide by taking tangents from the focal spot along the edge of the waveguide, as marked on the figure, and this gave $\theta=17^{\circ}$. We calculated the halfangle of the focal cone of the writing beam from the NA of the writing beam objective (0.55) and the index of GLS (2.4) to be $13^{\circ}$. Therefore a large portion, but not all, of the waveguide in Fig. 1(a) could have been formed by direct exposure to the writing beam. 
Focused fs pulses in sapphire can generate pressures of $\sim 10 \mathrm{TPa}$ and temperatures of $5 \times 10^{5} \mathrm{~K}$, converting material within the absorbing volume into plasma in a few fs. This results in the formation of a nanovoid surrounded by a shell of shock-affected material [36]. The shock-affected region had a teardrop shape, similar to GLS waveguides; however, the dimensions were on the order of $1 \mu \mathrm{m}$ [37]. Microexplosions have also been observed in fs-laser exposed soda-lime silicate glass using the transient lens method [38] and, in BK7 borosilicate glass, using atomic force microscopy [39]. Although the thermomechanical properties of sapphire are somewhat different from GLS, the glass transition temperatures of BK7 and GLS are very similar: 557 and $561^{\circ} \mathrm{C}$, respectively, as are the melting temperatures: 820 and $830^{\circ} \mathrm{C}$, respectively [40,41]. If microexplosions occur in GLS it is difficult to see how they alone create the largest waveguides, which had dimensions of $\sim 100 \times 300 \mu \mathrm{m}$, since the experiments in sapphire and BK7 glass indicate that microexplosions affect a limited volume. There was also no evidence of cracking or nanovoids in GLS. Shockwave generation without the formation of nanovoids has been attributed to slightly larger structures in fs-exposed glass; however, the affected region was still on the order of the focus waist $[38,42]$. In silica glass, shockwaves are known to produce Raman changes [43], which we did not observe. Therefore shockwave generation is unlikely, but it may explain the positive index change of a small section of region 2 , which immediately surrounds the exposed region.

It has been shown, by heating a soda-lime glass to various temperatures before irradiation, that a $250 \mathrm{kHz}$ fs laser can generate a modified region far larger than the focus waist by the cumulative heating effect of successive fs pulses [42]. The width of this modified region was up to $100 \mu \mathrm{m}$, which is very similar to the width of the largest GLS waveguides. Using various repetition rates, it has been shown that cumulative heating occurs at repetition rates greater than $200 \mathrm{kHz}$ in low-alkali boroaluminosilicate glass and alkali-free borosilicate glass but not in pure silica glass [44]. Since the bandgap and melting temperature of GLS is closer to that of low-alkali boro-aluminosilicate glass and alkali-free borosilicate glass than pure silica glass, it is reasonable to assume that the cumulative heating occurs in the waveguides we wrote in GLS using a repetition rate of $250 \mathrm{kHz}$.

It is difficult to determine which mechanism accounts for the structure of GLS waveguides. It is possible that all three mechanisms occur concurrently; however, the above discussion indicates that microexplosions can only play a minor role in the formation mechanism because they appear to modify a limited volume of solids. Therefore, either type IIA photosensitivity or cumulative heating or both together can account for the bulk of the waveguide structure. However, the effect of type IIA photosensitivity is probably strongest at the core of the waveguide, where the exposure intensity is highest. The effect of cumulative heating is probably strongest at the periphery of the waveguides, since it can penetrate well beyond the boundary of the writing beam. Therefore, the most probable formation mechanism is that the negative index change, in region 1, results from exposure to high intensities. The positive index changes in the inner and outer portions of region 2 are produced by exposure to lower intensities and by cumulative heating, respectively. The lack of information from the Raman measurements makes any further elucidation of the formation mechanism unproductive at this stage.

The linear shape of the B-type waveguides indicates that filamentation caused by self-focusing may have occurred. In earlier work on fs-laser written waveguides in GLS we calculated the loss of the set 1 and set 2 waveguides and found that the writing parameters for the minimum propagation loss of $1.47 \mathrm{~dB} / \mathrm{cm}$ were $0.36 \mu \mathrm{J}$ pulse energy and $50 \mu \mathrm{m} / \mathrm{s}$ scanning speed [20]. We also identified tunneling as the dominant nonlinear absorption mechanism by calculation of the Keldysh parameter.

\section{E. Spectral Broadening}

We measured the spectra of $1540 \mathrm{~nm}$ laser pulses, with a $50 \mathrm{~nm}$ full width at half-maximum (FWHM) and $200 \mathrm{fs}$ duration passing through all of the set 1 and 2 waveguides; however, we only observed significant spectral broadening in the waveguides shown in Figs. 1(a) and 1(b). We believe this was because the large cross-section area and index change of these waveguides allowed more light to be coupled into them. We calculated the power coupled into the waveguides from the output power from the waveguides and their loss.

Figure 6 shows the spectra of $1540 \mathrm{~nm}$ laser pulses, with a $200 \mathrm{fs}$ duration, after passing through $12 \mathrm{~mm}$ of GLS fs-laser written waveguide, as a function of the pulse energy coupled into the waveguide. The waveguide was written with pulse energies of $1.75 \mu \mathrm{J}$ and a translation speed of $200 \mu \mathrm{m} / \mathrm{s}$ and had a cross-section size of $\sim 100$ $\times 300 \mu \mathrm{m}$. The micrograph and guided mode at $633 \mathrm{~nm}$ of this waveguide can be seen in Fig. 1(a), and the guided mode at $1550 \mathrm{~nm}$ can be seen in Fig. 1(j). The spectrum of the laser beam before it had passed through the wave-

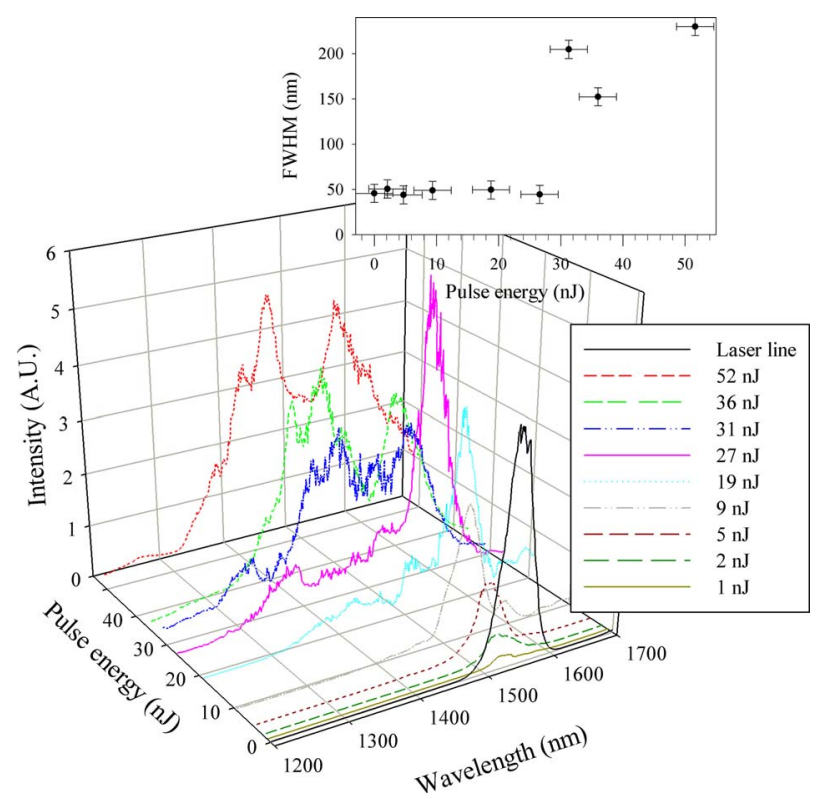

Fig. 6. (Color online) Spectra of $1540 \mathrm{~nm} 200 \mathrm{fs}$ laser beam coupled into a $12 \mathrm{~mm}$ GLS waveguide that was written with a pulse energy of $1.75 \mu \mathrm{J}$, as a function of input beam pulse energy. The insets show the FWHM as a function of pulse energy. 
guide is also shown. At pulse energies up to $4.7 \mathrm{~nJ}$ the output spectrum is very similar to the input spectrum except for a small blue shift in peak position. At $18.8 \mathrm{~nJ} /$ pulse the peak position is red shifted and at $31.3 \mathrm{~nJ} /$ pulse the spectrum changes dramatically from a peak still resembling the input spectrum to a very broad, almost flat spectrum. As the pulse energy is increased further, broad double-peak spectra are formed with one peak at roughly the same position as the input spectrum and the other at shorter wavelengths. The apparent decrease in signal-to-noise ratio with increasing pulse energy could be related to the complex topology of the refractive index profile, which could introduce different propagation phase shifts at different spatial points of the mode.

The inset of Fig. 6 shows the FWHM of the spectra in Fig. 6 as a function of pulse energy. Error bars for wavelength and pulse energy were estimated from the resolution of the OSA and the power meter, respectively. The figure indicates that the FWHM remains at a relatively constant $50 \mathrm{~nm}$ up to a pulse energy of $\sim 30 \mathrm{~nJ} / \mathrm{pulse}$ where there is a sudden increase in FWHM to $\sim 200 \mathrm{~nm}$. As the pulse energy is increased further, FWHM falls. This is attributed to the emergence of the slightly asymmetric double-peak structure. At pulse energies $>\sim 30 \mathrm{~nJ} /$ pulse the double-peak structure is evident with peaks at $\sim 1570$ and $1460 \mathrm{~nm}$.

Figure 7 shows the spectra of $1540 \mathrm{~nm}$ laser pulses with a $200 \mathrm{fs}$ duration, after passing through $12 \mathrm{~mm}$ of GLS fs written waveguide, as a function of the pulse energy coupled into the waveguide. The waveguide was written with a pulse energy of $1.26 \mu \mathrm{J}$ and a translation speed of $200 \mu \mathrm{m} / \mathrm{s}$ and had a cross-sectional size of $\sim 100 \times 220 \mu \mathrm{m}$. The micrograph and guided mode of this waveguide can be seen in Fig. 1(b). Comparisons with Fig. 6 show first that higher-pulse energies were coupled into this waveguide, which is attributed to the higher symmetry and maximum index change of this waveguide. Unlike

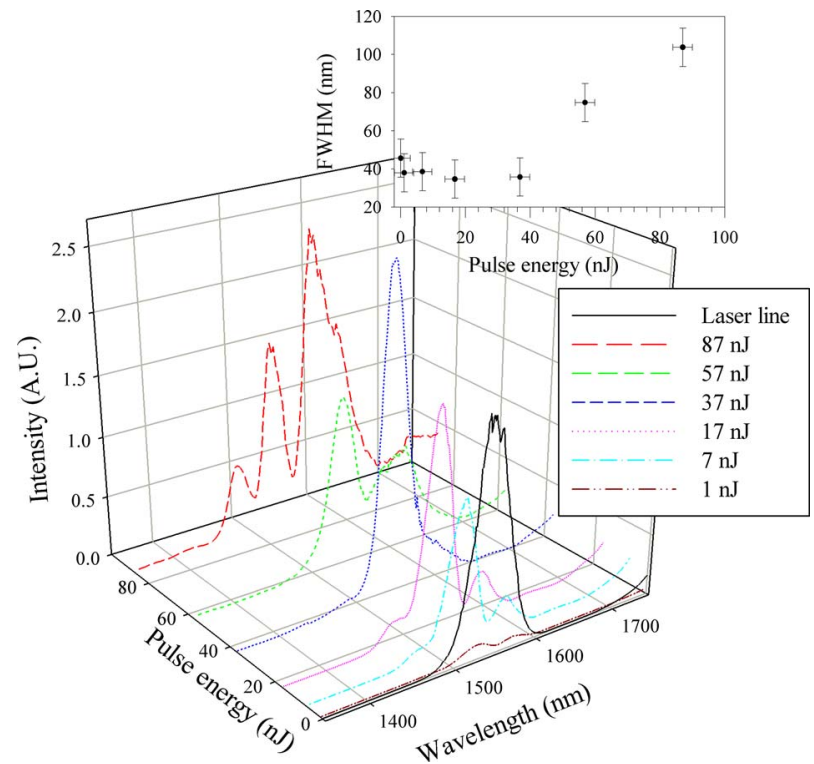

Fig. 7. (Color online) Spectra of $1540 \mathrm{~nm} 200 \mathrm{fs}$ laser beam coupled into a $12 \mathrm{~mm}$ GLS waveguide that was written with a pulse energy of $1.26 \mu \mathrm{J}$, as a function of input beam pulse energy. The insets show the FWHM as a function of pulse energy. the spectra in Fig. 6, a double-peak structure is evident at the lowest pulse energy of $1.1 \mathrm{~nJ}$. At $36.8 \mathrm{~nJ} /$ pulse the double-peak structure disappears then reappears at $56.9 \mathrm{~nJ} /$ pulse. At $87 \mathrm{~nJ} /$ pulse an asymmetric triple-peak structure is evident. The triple peak is indicative of a $2.5 \pi$ phase shift [45-47], and its weighting towards the long wavelength side of the pump is indicative of stimulated Raman scattering (SRS) [48,49].

The inset of Fig. 7 shows the FWHM as a function of pulse energy. Compared to Fig. 6 there is no significant peak shift of the input spectrum. The onset of broadening occurs at $\sim 60 \mathrm{~nJ} /$ pulse compared to $\sim 30 \mathrm{~nJ} /$ pulse in Fig. 6. The onset of broadening also appears to be more gradual and reaches a maximum of $\sim 100 \mathrm{~nm}$ compared to $\sim 220 \mathrm{~nm}$. The triple-peak spectrum at $87 \mathrm{~nJ} /$ pulse has peaks at $\sim 1530 \mathrm{~nm}, 1580 \mathrm{~nm}$, and $1480 \mathrm{~nm}$. The stronger broadening effect in the waveguide in Fig. 6 is attributed to its larger cross-section, which means that it could support more higher-order modes, that may take longer path lengths through the waveguide.

Broadening measurements in $50 \mu \mathrm{m}$ core diameter GLS fiber and single-mode, UV-written GLS waveguides were attempted, but broadening was not observed. This may have been because sufficient pulse energies could not be coupled into these waveguides to make a comparison with the broadening observed in fs-written waveguides. However, modeling indicates that $n_{2}$ should increase in chalcogenides that undergo photodarkening [50]. This leads to the intriguing possibility that broadening was only observed in the fs-written waveguides because $n_{2}$ was higher in the fs-written waveguide structure.

The number of peaks and the extent of the broadening in a self-phase modulation (SPM) broadened spectrum are dependent on the magnitude of the nonlinear phase shift and increase with it linearly [51]. The oscillatory structure of the broadened pulses in Figs. 6 and 7 are indicative of SPM. The maximum phase shift $\Phi_{\max }$ of an SPMbroadened pulse is given approximately by $[45,46]$

$$
\phi_{\max } \approx\left(M-\frac{1}{2}\right) \pi,
$$

where $M$ is the number of peaks in an SPM-broadened spectrum. Equation (1) and a comparison with other SPM-broadened spectra $[45,47]$ indicate that the doublepeak structure in Fig. 6 demonstrates a $1.5 \pi$ phase shift at $36 \mathrm{~nJ} /$ pulse, and the triple-peak structure in Fig. 7 demonstrates a $2.5 \pi$ phase shift at $87 \mathrm{~nJ} /$ pulse. These nonlinear phase shifts compare to a maximum phase shift of $1.6 \pi$ and $3.5 \pi$ in $2.03 \mathrm{~cm}$ [52] and $2.8 \mathrm{~cm}$ [48] long photodarkened planar $\mathrm{Ge}_{0.25} \mathrm{Se}_{0.75}$ glass waveguides, respectively. The spectral bandwidth of pulses broadened by $\operatorname{SPM}\left(\Delta \omega_{\mathrm{SPM}}\right)$ is given by [53]

$$
\Delta \omega_{\mathrm{SPM}}=\frac{\Delta \omega_{0} 2 \pi n_{2} P_{0} L}{\lambda A_{\mathrm{eff}}},
$$

where $\Delta \omega_{0}$ is the bandwidth of the input pulse. For the maximum pulse energy of $50 \mathrm{~nJ}$, used in Fig. 6, the bandwidth for pulses broadened by SPM is $\sim 100 \mathrm{~nm}$; however, the observed bandwidth of the broadened pulse was 
$\sim 200 \mathrm{~nm}$. This extra broadening is attributed to stimulated Raman scattering or an increase in $n_{2}$.

It may be possible to exploit the phase shift to realize a Mach-Zehnder interferometer switch. In this switch, guided light is split into two branches of a waveguiding structure and then recombined at an output in such a way that interference can occur. By inducing a $\pi$ or odd multiple of $\pi$ phase shift in one of the branches, the recombined beams can interfere destructively.

\section{CONCLUSIONS}

A formation mechanism is presented for fs-laser written waveguides in GLS glass, based on optical characterization and comparisons to previous work. Two different forms of waveguide are identified and referred to as A-type and B-type. A-type waveguides have a characteristic "teardrop" structure, with a central region (region 1) that has undergone a negative refractive index change, and an outer region (region 2) that has undergone a positive index change. B-type waveguides have a characteristic long narrow structure and are formed through filamentation. A-type waveguides form at pulse energies $>\sim 0.2 \mu \mathrm{J}$, and B-type waveguides form at pulse energies $<\sim 0.2 \mu \mathrm{J}$. The negative index change in region 1 results from exposure to high intensities. The positive index changes in the inner and outer portions of region 2 are produced by exposure to lower intensities and by cumulative heating, respectively. Only region 2 actively guides light. Waveguide transmission measurements indicate that, in A-type waveguides, intermolecular and intramolecular bond breaking occurs. However, in B-type waveguides only intermolecular bond breaking occurs. A maximum index change of 0.01 has been observed.

Spectral broadening from an initial FWHM of $50 \mathrm{~nm}$ to $200 \mathrm{~nm}$ has been demonstrated with a $1540 \mathrm{~nm}$, $200 \mathrm{fs}$ pulse at a pulse energy of $30 \mathrm{~nJ}$ in a waveguide written at a pulse energy of $1.75 \mu \mathrm{J}$. A change in peak position to $1580 \mathrm{~nm}$ was observed at $20 \mathrm{~nJ} /$ pulse. A maximum phase shift of $2.5 \pi$ has been demonstrated at a pulse energy of $88 \mathrm{~nJ} /$ pulse. The broadening has been attributed to self-phase modulation, with an asymmetry in some of the broadened spectra attributed to SRS. The high nonlinearity of GLS makes it a promising material for nonlinear optical devices. An interesting effect of this nonlinearity is the spectral broadening presented in this work.

\section{REFERENCES}

1. B. C. Stuart, M. D. Feit, A. M. Rubenchik, B. W. Shore, and M. D. Perry, "Laser-induced damage in dielectrics with nanosecond to subpicosecond pulses," Phys. Rev. Lett. 74, 2248-2257(1995).

2. L. Shah, J. Tawney, M. Richardson, and K. Richardson, "Self-focusing during femtosecond micromachining of silicate glasses," IEEE J. Quantum Electron. 40, 57-68 (2004).

3. F. Korte, S. Adams, A. Egbert, C. Fallnich, A. Ostendorf, S. Nolte, M. Will, J.-P. Ruske, B. Chichkov, and A. Tuennermann, "Sub-diffraction limited structuring of solid targets with femtosecond laser pulses," Opt. Express 7, 41-49 (2000).

4. S. Nolte, B. N. Chichkov, H. Welling, Y. Shani, K.
Lieberman, and H. Terkel, "Nanostructuring with spatially localized femtosecond laser pulses," Opt. Lett. 24, 914-916 (1999).

5. P. P. Pronko, S. K. Dutta, J. Squier, J. V. Rudd, D. Du, and G. Mourou, "Machining of sub-micron holes using a femtosecond laser at $800 \mathrm{~nm}$," Opt. Commun. 114, 106-110 (1995).

6. R. Osellame, S. Taccheo, G. Cerullo, M. Marangoni, D. Polli, R. Ramponi, P. Laporta, and S. De Silvestri, "Optical gain in $\mathrm{Er}-\mathrm{Yb}$ doped waveguides fabricated by femtosecond laser pulses," Electron. Lett. 38, 964-965 (2002).

7. E. N. Glezer, M. Milosavljevic, L. Huang, R. J. Finlay, T.-H. Her, J. P. Callan, and E. Mazur, "Three-dimensional optical storage inside transparent materials," Opt. Lett. 21, 2023-2025 (1996).

8. D. Ashkenasi, H. Varel, A. Rosenfeld, S. Henz, J. Herrmann, and E. E. B. Cambell, "Application of selffocusing of ps laser pulses for three-dimensional microstructuring of transparent materials," Appl. Phys. Lett. 72, 1442-1444 (1998).

9. J. Qiu, "Femtosecond laser induced microstructures in glass and application in micro-optics," Chem. Rec. 4, 50-58 (2004).

10. J. Qiu, K. Kojima, K. Miura, T. Mitsuyu, and K. Hirao, "Infrared femtosecond laser pulse induced permanent reduction of $\mathrm{Eu}^{3+}$ to $\mathrm{Eu}^{2+}$ in a fluorozirconate glass," Opt. Lett. 24, 786-788 (1999).

11. B. P. Nelson, K. J. Blow, P. D. Constantine, N. J. Doran, J. K. Lucek, I. W. Marshall, and K. Smith, "All-optical Gbit/s switching using nonlinear optical loop mirror," Electron. Lett. 27, 704-705 (1991).

12. K. Uchiyama, T. Morioka, and M. Saruwatari, "Polarization-independent wavelength conversion using nonlinear optical loop mirror," Electron. Lett. 31, 1862-1863 (1995).

13. K.-I. Kitayama, Y. Kimura, K. Okamoto, and S. Seikai, "Optical sampling using an all-fiber optical Kerr shutter," Appl. Phys. Lett. 46, 623-625 (1985).

14. E. Bricchi, J. D. Mills, P. G. Kazansky, B. G. Klappauf, and J. J. Baumberg, "Birefringent Fresnel zone plates in silica fabricated by femtosecond laser machining," Opt. Lett. 27, 2200-2202 (2002).

15. J. W. Chan, T. R. Huser, S. H. Risbud, J. S. Hayden, and D. M. Krol, "Waveguide fabrication in phosphate glasses using femtosecond laser pulses," Appl. Phys. Lett. 82, 2371-2373 (2003).

16. O. M. Efimov, L. B. Glebov, K. A. Richardson, E. Van Stryland, T. Cardinal, S. H. Park, M. Couzi, and J. L. Bruneel, "Waveguide writing in chalcogenide glasses by a train of femtosecond laser pulses," Opt. Mater. 17, 379-386 (2001).

17. J. Siegel, J. M. Fernandez-Navarro, A. Garcia-Navarro, V. Diez-Blanco, O. Sanz, J. Solis, F. Vega, and J. Armengol, "Waveguide structures in heavy metal oxide glass written with femtosecond laser pulses above the critical selffocusing threshold," Appl. Phys. Lett. 86, 121109 (2005).

18. C. Quémarda, F. Smektala, V. Coudercb, A. Barthélémyb, and J. Lucasa, "Chalcogenide glasses with high non linear optical properties for telecommunications," J. Phys. Chem. Solids 62, 1435-1440 (2001)

19. J. Requejo-Isidro, A. K. Mairaj, V. Pruneri, D. W. Hewak, M. C. Netti, and J. J. Baumberg, "Self refractive nonlinearities in chalcogenide based glasses," J. Non-Cryst. Solids 317, 241-246 (2003).

20. M. Hughes, W. Yang, and D. Hewak, "Fabrication and characterization of femtosecond laser written waveguides in chalcogenide glass," Appl. Phys. Lett. 90, 131113 (2007).

21. J. M. Senior, Optical Fiber Communications: Principles and Practice (Prentice Hall, 1992).

22. N. D. Psaila, R. R. Thomson, H. T. Bookey, S. Shen, N. Chiodo, R. Osellame, G. Cerullo, A. Jha, and A. K. Kar, "Supercontinuum generation in an ultrafast laser inscribed chalcogenide glass waveguide," Opt. Express 15, 15776-15781 (2007).

23. A. M. Streltsov and N. F. Borrelli, "Study of femtosecond- 
laser-written waveguides in glasses,” J. Opt. Soc. Am. B 19, 2496-2504 (2002).

24. A. Zoubir, M. Richardson, C. Rivero, A. Schulte, C. Lopez, K. Richardson, N. Ho, and R. Vallee, "Direct femtosecond laser writing of waveguides in $\mathrm{As}_{2} \mathrm{~S}_{3}$ thin films," Opt. Lett. 29, 748-750 (2004).

25. M. Hughes, H. Rutt, D. Hewak, and R. Curry, "Spectroscopy of vanadium (III) doped gallium lanthanum sulphide glass," Appl. Phys. Lett. 90, 031108 (2007).

26. K. Hirao and K. Miura, "Writing waveguides and gratings in silica and related materials by a femtosecond laser," J. Non-Cryst. Solids 239, 91-95 (1998).

27. S. R. Elliott, "A unified model for reversible photostructural effects in chalcogenide glasses," J. Non-Cryst. Solids 81, 71-98 (1986)

28. P. Hari, S. Guzel, T. Su, P. C. Taylor, P. L. Kuhns, W. G. Moulton, and N. S. Sullivan, "Photodarkening effect in glassy $\mathrm{As}_{2} \mathrm{~S}_{3}$ and $\mathrm{As}_{2} \mathrm{O}_{3}$,"J. Non-Cryst. Solids 326, 199-204 (2003).

29. G. Pfeiffer, C. J. Brabec, S. R. Jefferys, and M. A. Paesler, "Structural models of glassy $\mathrm{As}_{2} \mathrm{~s}_{3}$-intermediate-range order and photostructural changes," Phys. Rev. B 39, 12861-12871 (1989).

30. G. Pfeiffer, M. A. Paesler, and S. C. Agarwal, "Reversible photodarkening of amorphous arsenic chalcogens," J. NonCryst. Solids 130, 111-143 (1991)

31. K. Tanaka, "Reversible photoinduced change in intermolecular distance in amorphous $\mathrm{As}_{2} \mathrm{~S}_{3}$ network," Appl. Phys. Lett. 26, 243-245 (1975).

32. V. K. Tikhomirov, A. B. Seddon, K. Asatryan, T. V. Galstian, and R. Vallee, "The role of van der Waals bonding in photosensitivity of chalcogenide glasses," J. Non-Cryst. Solids 326, 205-208 (2003).

33. D. K. Y. Low, H. Xie, Z. Xiong, and G. C. Lim, "Femtosecond laser direct writing of embedded optical waveguides in aluminosilicate glass," Appl. Phys. A 81, 1633-1638 (2005).

34. M. V. Bazylenko, D. Moss, and J. Canning, "Complex photosensitivity observed in germanosilica planar waveguides," Opt. Lett. 23, 697-699 (1998).

35. C. Pappas and S. Pissadakis, "Periodic nanostructuring of Er/Yb-codoped IOG1 phosphate glass by using ultraviolet laser-assisted selective chemical etching," J. Appl. Phys. 100, 114308 (2006).

36. S. Juodkazis, K. Nishimura, S. Tanaka, H. Misawa, E. G. Gamaly, B. Luther-Davies, L. Hallo, P. Nicolai, and V. T. Tikhonchuk, "Laser-induced microexplosion confined in the bulk of a sapphire crystal: Evidence of multimegabar pressures," Phys. Rev. Lett. 96, 166101 (2006).

37. E. G. Gamaly, S. Juodkazis, K. Nishimura, H. Misawa, and B. Luther-Davies, "Laser-matter interaction in the bulk of a transparent solid: Confined microexplosion and void formation," Phys. Rev. B 73, 214101 (2006).

38. M. Sakakura and M. Terazima, "Initial temporal and spatial changes of the refractive index induced by focused femtosecond pulsed laser irradiation inside a glass," Phys. Rev. B 71, 024113 (2005).
39 E. N. Glezer and E. Mazur, "Ultrafast-laser driven microexplosions in transparent materials," Appl. Phys. Lett. 71, 882-884 (1997).

40. P. E. Dyer, S. M. Maswadi, C. D. Walton, M. Ersoz, P. D. I. Fletcher, and V. N. Paunov, "157-nm laser micromachining of N-BK7 glass and replication for microcontact printing," Appl. Phys. A 77, 391-394 (2003).

41. Y. D. West, T. Schweizer, D. J. Brady, and D. W. Hewak, "Gallium lanthanum sulphide fibers for infrared transmission," Fiber Integr. Opt. 19, 229-250 (2000).

42. M. Sakakura, M. Shimizu, Y. Shimotsuma, K. Miura, and K. Hirao, "Temperature distribution and modification mechanism inside glass with heat accumulation during $250 \mathrm{kHz}$ irradiation of femtosecond laser pulses," Appl. Phys. Lett. 93, 231112 (2008).

43. J. W. Chan, T. R. Huser, S. H. Risbud, and D. M. Krol, "Modification of the fused silica glass network associated with waveguide fabrication using femtosecond laser pulses," Appl. Phys. A 76, 367-372 (2003).

44. S. M. Eaton, H. B. Zhang, and P. R. Herman, "Heat accumulation effects in femtosecond laser-written waveguides with variable repetition rate," Opt. Express 13, 4708-4716 (2005).

45. G. P. Agrawal, Nonlinear Fiber Optics (Academic, 2001).

46. R. Cubeddu, R. Polloni, C. A. Sacchi, and O. Svelto, "Selfphase modulation and "rocking" of molecules in trapped filaments of light with picosecond pulses," Phys. Rev. A 2 , 1955-1963 (1970).

47. R. H. Stolen and C. Lin, "Self-phase-modulation in silica optical fibers," Phys. Rev. A 17, 1448-1453 (1978).

48. S. Spalter, G. Lenz, R. E. Slusher, H. Y. Hwang, J. Zimmermann, T. Ktsufuji, S. W. Cheong, and M. E. Lines, "Highly nonlinear chalcogenide glasses for ultrafast all optical switching in optical TDM communication systems," in Optical Fiber Communication Conference 2000 (Optical Society of America, 2000), p. 137.

49. D.-P. Wei, T. Galstian, I. Smolnikov, V. Plotnichenko, and A. Zohrabyan, "Spectral broadening of femtosecond pulses in a single-mode As-S glass fiber," Opt. Express 13, 2439-2443 (2005).

50. K. Petkov and P. J. S. Ewen, "Photoinduced changes in the linear and non-linear optical properties of chalcogenide glasses," J. Non-Cryst. Solids 249, 150-159 (1999).

51. J. K. Kim, "Investigation of high-nonlinearity glass fibers for potential applications in ultrafast nonlinear fiber devices," Ph.D. dissertation (Virginia State University, 2005).

52. S. Spälter, H. Y. Hwang, J. Zimmermann, G. Lenz, T. Katsufuji, S.-W. Cheong, and R. E. Slusher, "Strong selfphase modulation in planar chalcogenide glass waveguides," Opt. Lett. 27, 363-365 (2002).

53. P. V. Mamyshev, "All-optical data regeneration based on self-phase modulation effect," in 24th European Conference on Optical Communication, (IEEE, 1998), p. 475. 